\title{
Self-Consistent Evolution of Tissue Damage Under Stress Wave Propagation
}

\author{
M. Strauss, Y. Kaufman, M. Sapir,
} P. Amendt, R. A. London, M. E. Glinsky

This paper was prepared for submittal to the

Photonics West' 99 Symposium

San Jose, CA

January 23-29, 1999

January 14, 1999

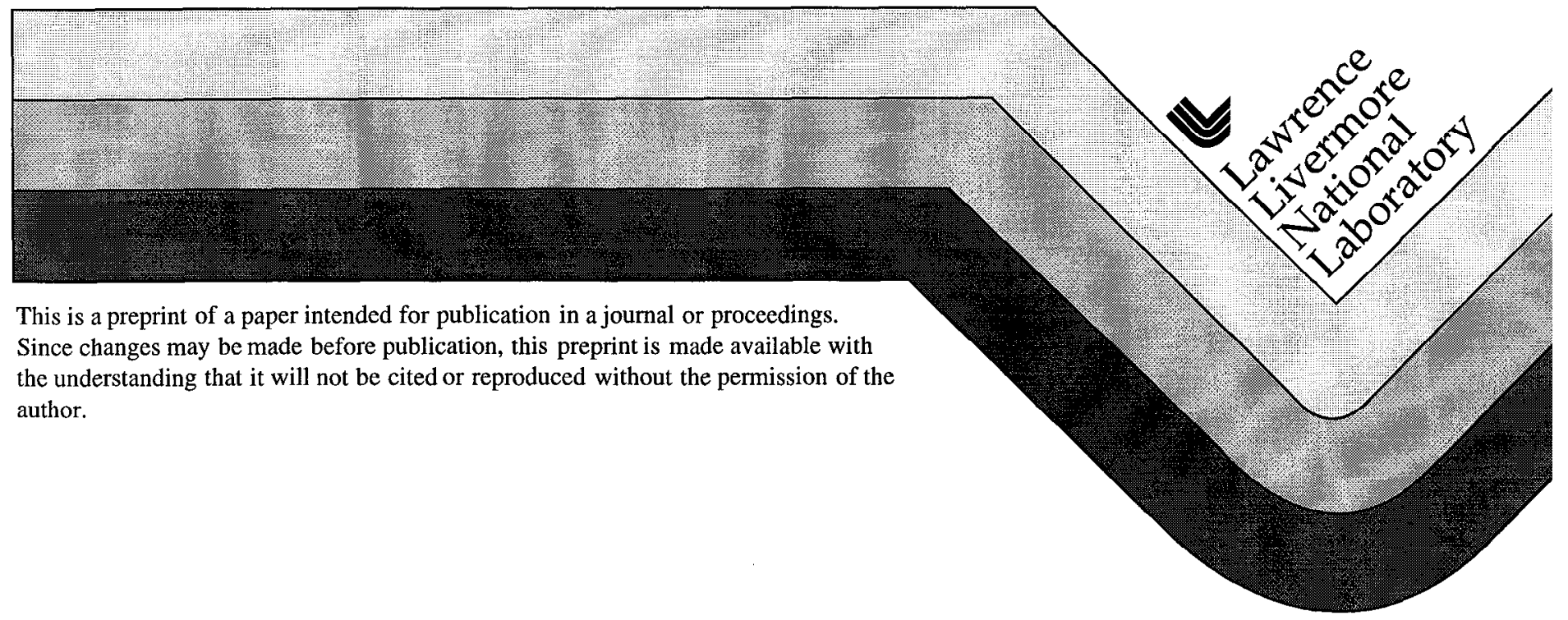




\section{DISCLATMER}

This document was prepared as an account of work sponsored by an agency of the United States Government. Neither the United States Government nor the University of California nor any of their employees, makes any warranty, express or implied, or assumes any legal liability or responsibility for the accuracy, completeness, or usefulness of any information, apparatus, product, or process disclosed, or represents that its use would not infringe privately owned rights. Reference herein to any specific commercial product, process, or service by trade name, trademark, manufacturer, or otherwise, does not necessarily constitute or imply its endorsement, recommendation, or favoring by the United States Government or the University of California. The views and opinions of authors expressed herein do not necessarily state or reflect those of the United States Government or the University of California, and shall not be used for advertising or product endorsement purposes. 


\title{
Self-consistent evolution of tissue damage under stress wave propagation
}

\author{
Moshe Strauss, Yitzhak Kaufman, Micha Sapir, \\ Peter Amendt ${ }^{*}$, Richard A. London ${ }^{*}$ and Michael E. Glinsky ${ }^{+}$ \\ Nuclear Research Center, Negev, P.O.Box 9001, Beer Sheva, Israel \\ *Lawrence Livermore National Laboratory, Livermore, CA 94550 \\ ${ }^{+}$Shell E \& P Technology Co. Houston, TX 77025
}

\begin{abstract}
Laser-initiated stress waves are reflected from tissue boundaries, thereby inducing tensile stresses, which are responsible for tissue damage. A self-consistent model of tissue failure evolution induced by stress wave propagation is considered. The failed tissue is represented by an ensemble of spherical voids and includes the effect of nucleation, growth and coalescence of voids under stress wave tension. Voids nucleate around impurities and grow according to an extended Rayleigh model that includes the effects of surface tension, viscosity and acoustic emission at void collapse. The damage model is coupled self-consistently to a one-dimensional planar hydrodynamic model of stress waves generated by a short pulse laser. We considered the problem of a bipolar wave generated by a short pulse laser absorbed on a free boundary of an aqueous system. The propagating wave includes a tensile component, which interacts with the impurities of exponential distribution in dimension, and an ensemble of voids is generated. For moderate impurity density $\left(\sim 10^{8} \mathrm{~cm}^{-3}\right)$ void growth reduces the tensile wave component and causes the pressure to oscillate between tension and compression. For low impurity density $\left(\sim 10^{6} \mathrm{~cm}^{-3}\right)$ the bubbles grow on a long time scale $(5-10 \mu \mathrm{sec})$ relative to the wave interaction time $(-100 \mathrm{nsec})$. At later times the growing bubbles interact with each other causing pressure oscillations and delay the system from reaching the 1 bar ambient compression pressure. This effect increases considerably the bubble lifetime consistent with experiments. At the collapse stage small bubbles collapse earlier and induce pressures, which reduce the collapse time of the larger bubbles.
\end{abstract}

Keywords: stress wave, material failure, bubble, void, simulation

\section{INTRODUCTION}

In many medical applications laser-initiated stress waves induce tensile waves, which are responsible for tissue damage. Tensile stresses are generated from laser deposition on tissue boundaries and from diffraction of stress waves while propagating inside tissues. ${ }^{1,2.3}$ The purpose of this paper is to study the mechanisms involved in the process of tissue damage. The damage can be divided to the following stages: nucleation, growth and coalescence. ${ }^{4,5}$ The damage starts by the nucleation of an ensemble of voids around existing impurities induced by tensile stresses. The growing voids can affect the stress wave propagation. At later times the voids can interact with each other through the system pressure affecting their dynamics and lifetime. ${ }^{1}$ Thus a selfconsistent model coupling stress wave propagation and void evolution is required.

We consider a bipolar wave generated on a free boundary by a short pulse laser in an aqueous system. The system is described by a one-dimensional hydrodynamic code coupled to an ensemble of impurities of exponential shape in size and nucleating a distribution of voids. The 
voids grow as spherical bubbles according to an extended Rayleigh model based on the KirkwoodBethe hypothesis. ${ }^{6.7}$ Two typical densities are defined in the liquid: an average density between the voids and the liquid affecting mainly the hydrodynamic motion, and a local density of the liquid affecting the thermodynamic variables as pressure through the equation of state (EOS). The evolving voids reduce the tension in the system and may cause even local compression, which induces pressure oscillation and affects the void growth. The pressure in the system is highly sensitive to density variations. Thus the growing bubbles through density variations and pressure changes interact with each other long before they reach the coalescence stage.

We find that for moderate impurity densities $\left(\sim 10^{8} \mathrm{~cm}^{-3}\right)$ the void growth reduces the stress wave tension and imposes oscillation between tension and compression. For low impurity density $\left(-10^{6} \mathrm{~cm}^{-3}\right)$ voids growing on a microsecond time scale interact with each other through the variation in the density and pressure. This interaction delays the system from reaching the 1 bar ambient compression pressure and increases the bubble lifetimes by almost a factor of 2 . This result is consistent with experimental results obtained by Paltauf and Schmidt-Kloiber. ${ }^{1}$ We further find that small bubbles collapse earlier and cause an increase in pressure oscillations and a reduction in the larger bubble lifetimes.

The paper is organized as follows: Section 2 discusses the evolution model of an ensemble of voids. Section 3 presents the hydrodynamics of the system in the presence of evolving voids. Section 4 presents the computational results, and concluding remarks are given in Section 5.

\section{EVOLUTION MODEL OF AN ENSEMBLE OF VOIDS}

We consider a short pulse laser absorbed on a free boundary of an aqueous liquid. A bipolar stress wave is generated from an instantaneous deposition of the laser energy and includes compression and tensile components. The tensile component acts on the system and generates a distribution of spherical bubbles or voids around the embedded ensemble of impurities. ${ }^{4,5}$ In the following we consider the nucleation, growth and coalescence of the ensemble of voids. In a real tissue the growing voids reduce the tissue strength and may coalesce to produce a complete fracture in the tissue.

\subsection{Nucleation}

We consider a distribution of impurities acting as nucleation centers. The impurities have an exponential distribution in dimension, ${ }^{4}$

$$
n(R)=\frac{n_{0}}{R_{I}} \exp \left(-R / R_{l}\right),
$$

where $n \cdot d R$ is the number of impurities per unit volume with radius $R$ in the range $d R, n_{0}$ is impurity number density and $R_{J}$ is a dimensional parameter of the distribution. In general it is possible to consider other types of impurity distribution. Typical values are $n_{0} \sim 10^{5}-10^{8} \mathrm{~cm}^{-3}$ and $R \sim 0.1-2 \mu \mathrm{m}$. The surface tension pressure limits the nucleation threshold $20 / R$, where $\sigma$ is the surface tension parameter. In water $\sigma=70 \mathrm{erg} / \mathrm{cm}^{2}$ and for tensions larger then 10 bar voids nucleate around impurities with radius $R>0.15 \mu \mathrm{m}$. 


\subsection{Growth}

The voids grow as spherical bubbles around the impurities according to an extended Rayleigh model based on the Kirkwood-Bethe hypothesis. The ordinary differential equation for the bubble radius $R$ is, $1,8,9$

$$
R \dot{U}(1-M)+\frac{2}{3} U^{2}\left(1-\frac{M}{3}\right)=\Delta H \cdot(1+M)+\frac{R}{C_{s}} \Delta \dot{H}(1-M)
$$

where $U=\dot{R}$ is the bubble boundary velocity, $M=U / C_{s}$ is the Mach number and $C_{s}$ is the adiabatic sound speed. The enthalpy difference $\Delta H$ is given by,

$$
\Delta H=H(R)-H_{a}=\int_{P_{a}}^{P(R)} \frac{d P}{\rho}
$$

where the enthalpy $H(R)$ and the pressure $P(R)$ are on the bubble boundary; the enthalpy $\mathrm{H}_{\mathrm{a}}$ and the ambient pressure $P_{a}$ are far from the bubble boundary. The enthalpies can be written as,

$$
H(R)=\left(\varepsilon+\frac{P}{\rho}\right)_{r=R} \quad, H_{a}=\left(\varepsilon+\frac{P}{\rho}\right)_{r=r_{a}}, \quad,
$$

where $\varepsilon$ is the energy per unit mass and $\rho$ the density. The ambient pressure $P_{a}$ is taken as the local and time dependent pressure obtained from the hydrodynamic simulation. The pressure $P(R)$ on the bubble boundary depends on the pressure $\mathrm{P}_{\mathrm{b}}$ inside the bubble, the surface tension $\sigma$, and the viscosity $\eta$,

$$
P(R)=P_{b}\left(R, S_{b}\right)-\frac{2 \sigma}{R}-4 \eta \frac{U}{R}
$$

where for water $\eta=10^{-2} \mathrm{erg} \cdot \mathrm{sec} / \mathrm{cm}^{3}$. The surface tension and the viscosity impose a threshold pressure for void nucleation.

The first term on the right of eq.(2) depends on the difference between the pres:ure inside the bubble and the ambient pressure. For small density changes one obtains from eq.(3), $\Delta H \approx\left(P(R)-P_{a}\right) / \rho$ and by inserting it into eq. (2) we get a more familiar form of the extended Rayleigh equation, but less accurate. The second term on the right depends on the rate of change of the enthalpy and presents the acoustic emission by the bubble. Equation (2) can be applied for large acoustic emission with Mach number up to $\mathrm{M} \sim 1$.

When the bubble grows under tension, the pressure inside the bubble is reduced almost to zero. But in the collapse stage of the bubble its volume is limited by the compressibility of the impurity inside the bubble. This situation can be presented by applying inside the bubble a Maxwell constructed equation of state (EOS) ${ }^{10}$ In the region outside the bubble where tensile wave can exist a Van der Waals EOS is selected. ${ }^{10}$ In the following simulations EOS of water is used inside and outside the bubble. The expansion of the bubble is adiabatic. Thus adiabats can be generated for the inside and outside of the bubble, based on the initial condition after the heating of the svstem by the 
laser. The adiabats are presented for a given entropy $S_{0}$ in a table of pressure, temperature and energy per unit mass as a function density. The adiabats are used to describe the bubble expansion in eq.(2).

\section{$\underline{2.3 \text { Coalescence }}$}

The growing bubbles interact with each other by changing the liquid density or pressure around them. When the void area can be linked together a fracture in the system is obtained. According to the percolation theory this happens for a relative area of $\sigma_{A C}=0.4 .^{12}$ We apply a simplified criterion for fracture according to the relative void volume. We assume that the void volume is related to the void area as a $3 / 2$ power and select the fracture criteria for a relative void volume larger than the value $\sigma_{V C}=0.25$.

\section{SELF CONSISTENT HYDRODINAMICS AND DAMAGE EVOLUTION}

In the hydrodynamic model, the liquid system including the voids behaves as a porous material with an average density $\rho_{a v}$ that differs from the local liquid density $\rho$. The two densitics are related by

$$
\rho=\rho_{a v} \frac{\left(1-\sigma_{v 0}\right)}{\left(1-\theta_{v}\right)},
$$

where $\sigma_{v}$ is the local relative void volume, $\sigma_{v 0}$ is the initial relative volume of the impurities and at $t=0$ we have $\rho=\rho_{a v}$. The hydrodynamic computations are done with $\rho_{a v}$, while the thermodynamic quantities as pressure, heat capacity and sound speed are calculated using the EOS according to the local fluid density $\rho$. The increase in the void volume causes an increase in the liquid density $\rho$ relative to the average density $\rho_{a v}$, which reduces the tension in the system. This couples the hydrodynamic motion to the void evolution. The sensitivity of pressure to density variations is high while other thermodynamic quantities, as heat capacity and sound speed, are weakly sensitive to density changes.

The ensemble of impurities is represented by $N$ initial radii $R_{i}(0)$ at time $t=0$. The radii $R_{i}(t)$ evolve according to the extended Rayleigh model as in eq.(2), depending on the local hydrodynamic pressure $P_{a}$. Any other void radius $R(t)$ in the ensemble is obtained by linear interpolation from the calculated $R_{i}(t)$. This procedure is used to calculate the relative void volume $\sigma_{V}(t)$. The thermodynamic variables such as pressure, temperature and energy density are calculated inside and outside the bubbles based on the EOS adiabats. The adiabat table for the pressure, density and energy density are obtained for various densities in the beginning of the computation from knowing the initial entropies inside and outside the bubble.

At the end of every time step and in every spatial hydrodynamic zone, the bubble radii and the relative void volume $\sigma_{V}(t)$ are calculated based on the local hydrodynamic pressure In the following time step $\sigma_{V}(t)$ is used to transform $\rho_{a v}$ to the liquid density $\rho$ according to eq.(6). 
While the density $\rho$ and the temperature $T$ are included in the iterations, $\sigma_{V}(t)$ is taken from the previous time step to avoid numerical fluctuations.

In the coalescence stage when $\sigma_{V}(t)>G_{V C}(t)$, the system is locally fractured and the bubbles return to their initial distribution around the impurities. When compression is attained, the fracture can heal. Voids can be regenerated when tensile stresses are returned.

\section{RESULTS AND DISCUSSION}

We consider here the simulation of a propagating stress, wave interacting with an ensemble of spherical voids that nucleate and grow around a distribution of impurities. ${ }^{1}$ A short pulse laser is absorbed on a free boundary of an aqueous liquid system and a propagating bipolar stress wave is generated including compression and tension components. The laser energy is deposited in the system with an absorption length $l=100 \mu \mathrm{m}$, heating the system from its initial temperature $T_{0}=20^{\circ} \mathrm{C}$. The initial ambient pressure of the system is 1 bar. The tensile component interacts with the ensemble of impurities and nucleates a distribution of growing voids. We consider an exponential distribution of impurities of the form of eq.(1), where the parameter $R_{I}$ $=0.5 \mu \mathrm{m}$. We select five groups of impurities $(n=5)$ with initial radii $R_{i}=0.3,0.5,0.7,1.0,1.5 \mu \mathrm{m}$ and develop them as spherical voids or bubbles in the pressure field of the propagating stress wave. The growth of other bubbles is obtained by interpolation from the selected five bubble groups. We find that the results are not sensitive to an increase in the number of impurity radii $R_{i}$. We use a Van der Waals type equation of state (EOS) of water for the liquid system including tension. ${ }^{10}$. This EOS is consistent with the NBS Steam Tables ${ }^{12}$ with a sound speed $c_{s}=1.49 \cdot 10^{5} \mathrm{~cm} / \mathrm{sec}$ and Gruneisen coefficient $I=a+b T$ for temperature $T$ below $100{ }^{\circ} \mathrm{C}$, where $a=0.053, b=0.0037, T$ in ${ }^{\circ} \mathrm{C}$ and $C_{v}=3.8 \mathrm{~J} /\left(\mathrm{g}^{\circ} \mathrm{C}\right){ }^{1}$. The EOS applied in the Rayleigh expansion model [eq.(2)] for the bubble interior is a Maxwell constructed water EOS, ${ }^{10}$ with almost zero pressure under tension above its initial volume and almost incompressible under compression below its initial volume.

\subsection{Short time scale evolution}

Figure (1a) shows the stress wave pressure as a function of distance $x$ from the free surface at times 50,150,250 nsec and for very low impurity concentration $n_{0} \approx 0$. The bipolar wave is propagating almost unaltered through the system. Figure $(1 \mathrm{~b})$ considers the stress wave propagation for a moderate impurity density $n_{0}=10^{8} \mathrm{~cm}^{-3}$. As the wave is propagates inside the ystem the void growth imposes a reduction in the tensile component and causes the pressure to oscillate between tension and compression. The pressure oscillations are related to the density variation while the voids are changing their volume.

Figure (2) shows a time dependent comparison of the stress wave pressure for densities $n_{0}=0,10^{5}, 10^{8}, 10^{9} \mathrm{~cm}^{-3}$ at $x=220 \mu \mathrm{m}$. The increase in impurity density $n_{0}$ to $n_{0}=10^{9} \mathrm{~cm}^{-3}$ almost completely screens the tensile component on the time scale of stress wave propagation. The screening of the stress wave for densities below $n_{0}=10^{5} \mathrm{~cm}^{-3}$ is small. 

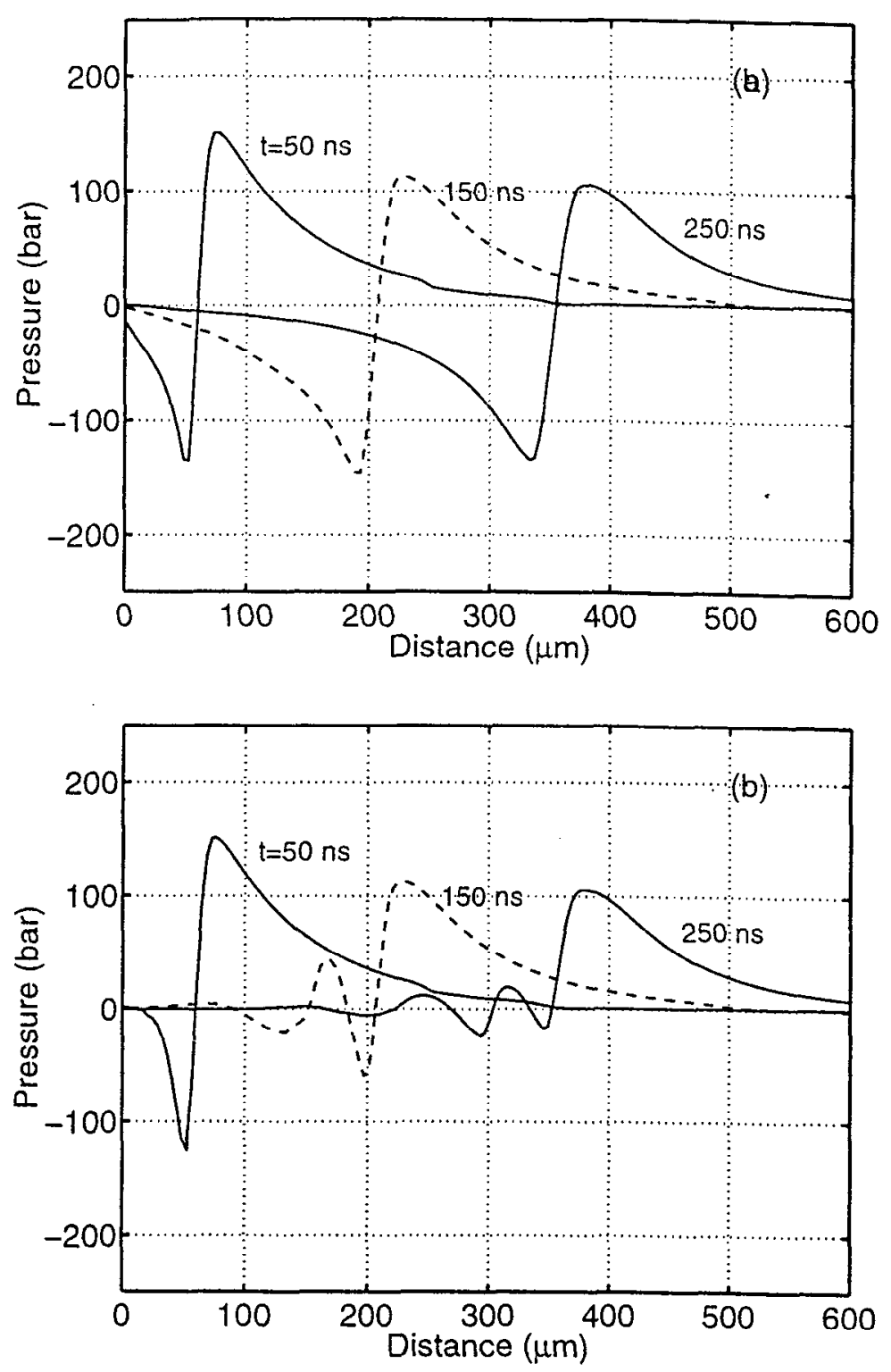

Fig. 1. Pressure vs distance for (a) impurity density $n_{0} \approx 0$ and (b) $n_{0}=10^{8} \mathrm{~cm}^{-3}$ at times $\mathrm{t}=50,150,250 \mathrm{~ns}$. 


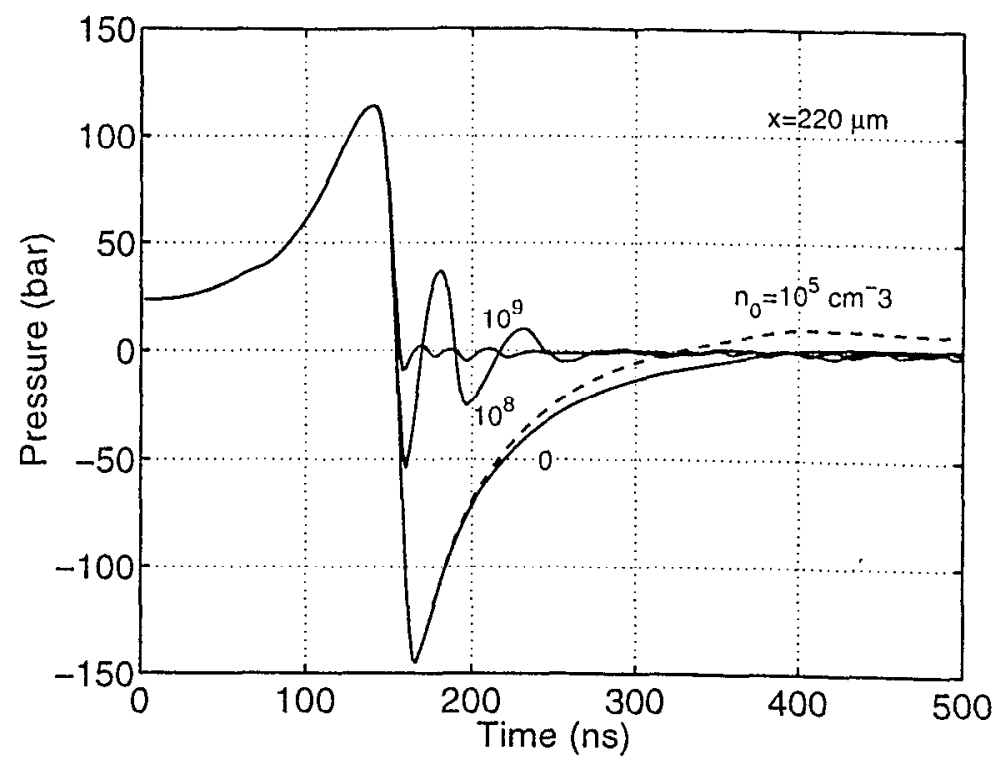

Fig. 2. Pressure vs time for impurity densities $n_{0}=0,10^{5}, 10^{8}, 10^{9} \mathrm{~cm}^{-3}$ at a distance of $220 \mu \mathrm{m}$ from the free surface.

Figure (3) shows the relative bubble volume $\sigma_{V}$ normalized to its maximum value as a function of distance from the free boundary for impurity densities $n_{0}=0,10^{6}, 10^{7}, 10^{8} \mathrm{~cm}^{-3}$ at time $500 \mathrm{~ns}$. As the impurity density increases the bubble volume is further reduced toward the inside part of the system. This is caused by the screening of the tensile component of the propagating stress wave as the impurity density increases.

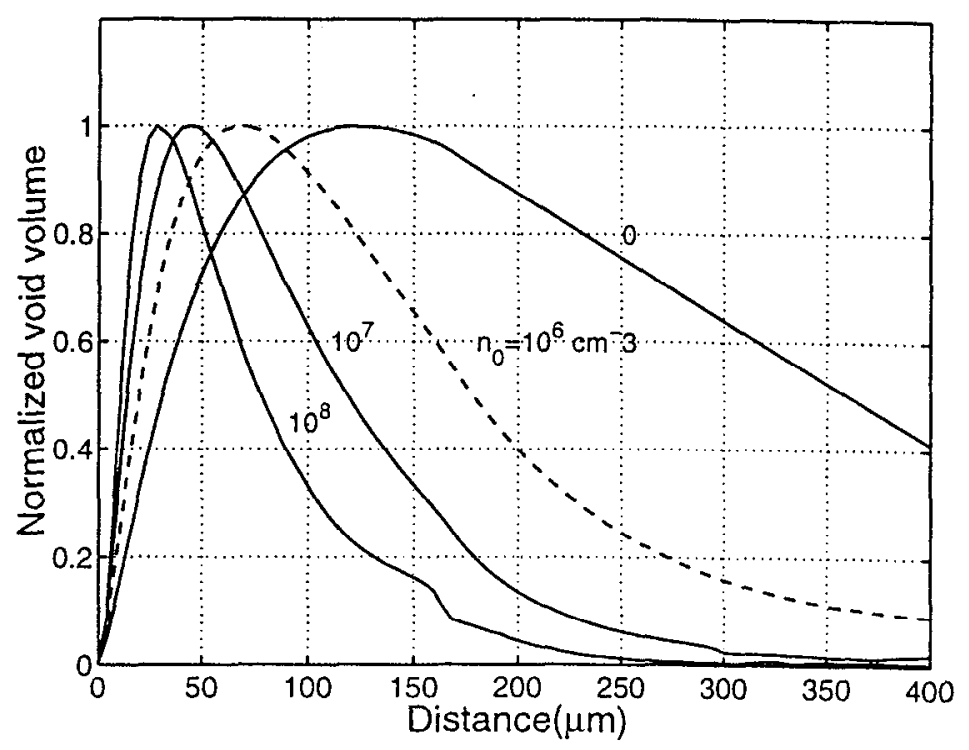

Fig. 3. The bubble relative volume normalized to its maximum value vs distance for impurity densities $n_{0}=0,10^{6}, 10^{7}, 10^{8} \mathrm{~cm}^{-3}$ at time $500 \mathrm{~ns}$. 


\subsection{Long time scale evolution}

In this section we consider evolution on time scales of the order of the bubble lifetimes. Figure (4) shows the bipolar wave pressure and a selection of bubble radii with initial values of $R_{i}=0.3,0.5,0.7,1.0 .1 .5 \mu \mathrm{m}$ as a function of time for very low impurity concentration, $n_{0} \approx 0$ for a distance $x=220 \mu \mathrm{m}$. The bubbles grows without interaction and collapse on a time scale of about 6 $\mu$ sec. The collapse is due to the $l$ bar ambient compression pressure in the aqueous system. The collapse time is similar for the various radii and weakly depends on the propagation distance $\mathrm{x}$.

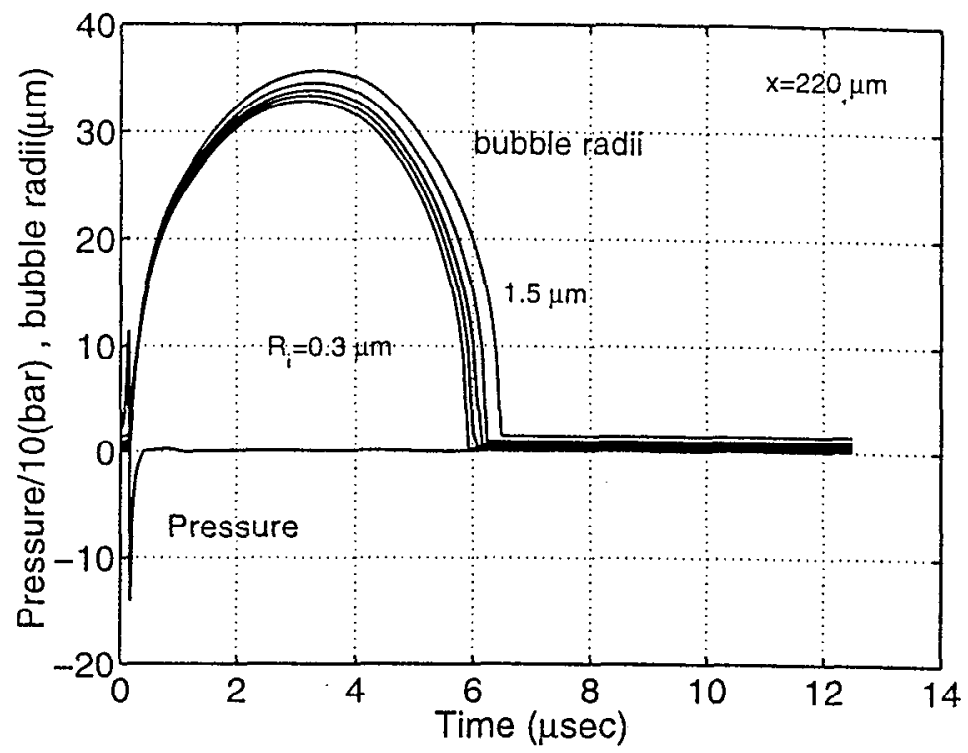

Fig. 4. Pressure $/ 10$ and bubble radii with initial values $R_{i}=0.3,0.5,0.7,1.0,1.5 \mu m$ vs time for impurity densities $n_{0} \approx 0$ and distance $\mathrm{x}=220 \mu \mathrm{m}$.

Figures (5a) and (5b) show mutual influence of the stress wave and bubble growth for impurity density $n_{0}=10^{6} \mathrm{~cm}^{-3}$ at distances of $x=220,340 \mu \mathrm{m}$. The growing bubbles interact with each other causing pressure oscillations. This prevents the system from reaching 1 bar compression pressure and bubble collapse is delayed. Bubble lifetime is delayed in fig. (5a) up to $10 \mu \mathrm{sec}$ which is almost by a factor of 2 longer than the non-interacting case in fig. (4). This result is consistent with the experimental results. ${ }^{1}$ Bubbles with initial smaller radii collapse earlier and there is a larger spread in bubble collapse time for the various bubble radii. In fig. (5b) we find that for larger distances, as $x=340 \mu \mathrm{m}$, the collapse times of the smaller bubbles is reduced and the spread of the collapse times for various radii is increased.

To follow the oscillatory nature of the pressure in the system while the bubbles are growing and collapsing, we show in fig. (6a) the pressure on a larger scale (bar). Pressure oscillations are seen as a function of time preventing the system from reaching the 1 bar ambient pressure thus delaying the bubbles from collapse. As the small bubbles collapse, the oscillations in pressure increase and tend to reduce the lifetime of larger bubble. The collapse of the larger bubbles induces even higher pressure oscillations. In fig.(6b) we present for clarity in an expanded time axis the pressure signal and the initial stage of the bubbles expansion as a function of time. The bipolar nature of the stress wave and the initial spread in the bubble radius evolution is presented. 

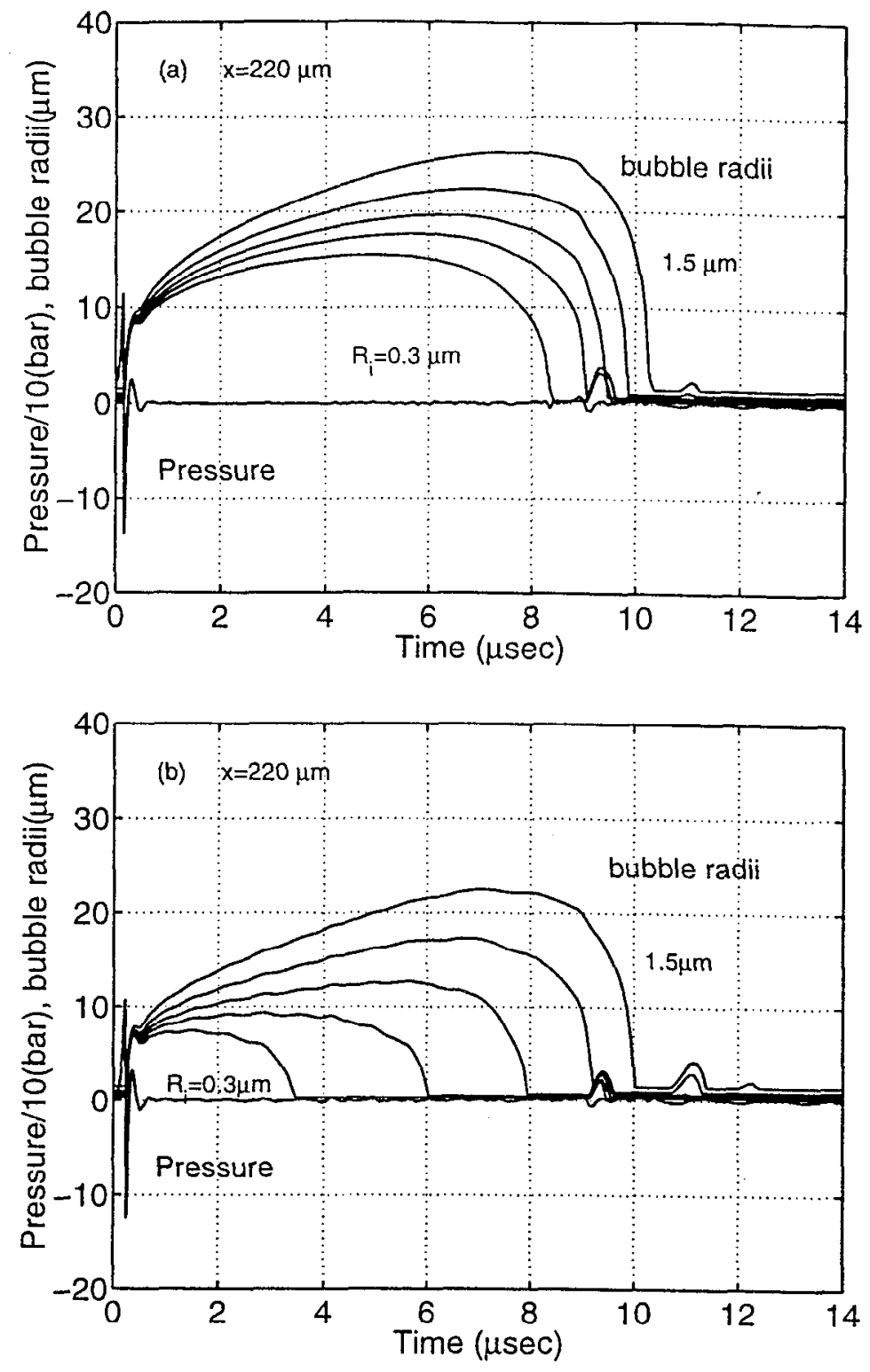

Fig. 5. Pressure $/ 10$ and bubble radii with initial values $R_{i}=0.3,0.5,0.7,1 ., 1.5 \mu \mathrm{m}$ vs time for impurity densities $n_{0}=10^{6} \mathrm{~cm}^{-3}$ for distance of (a) $x=220 \mu \mathrm{m}$ and (b) $x=340 \mu \mathrm{m}$. 

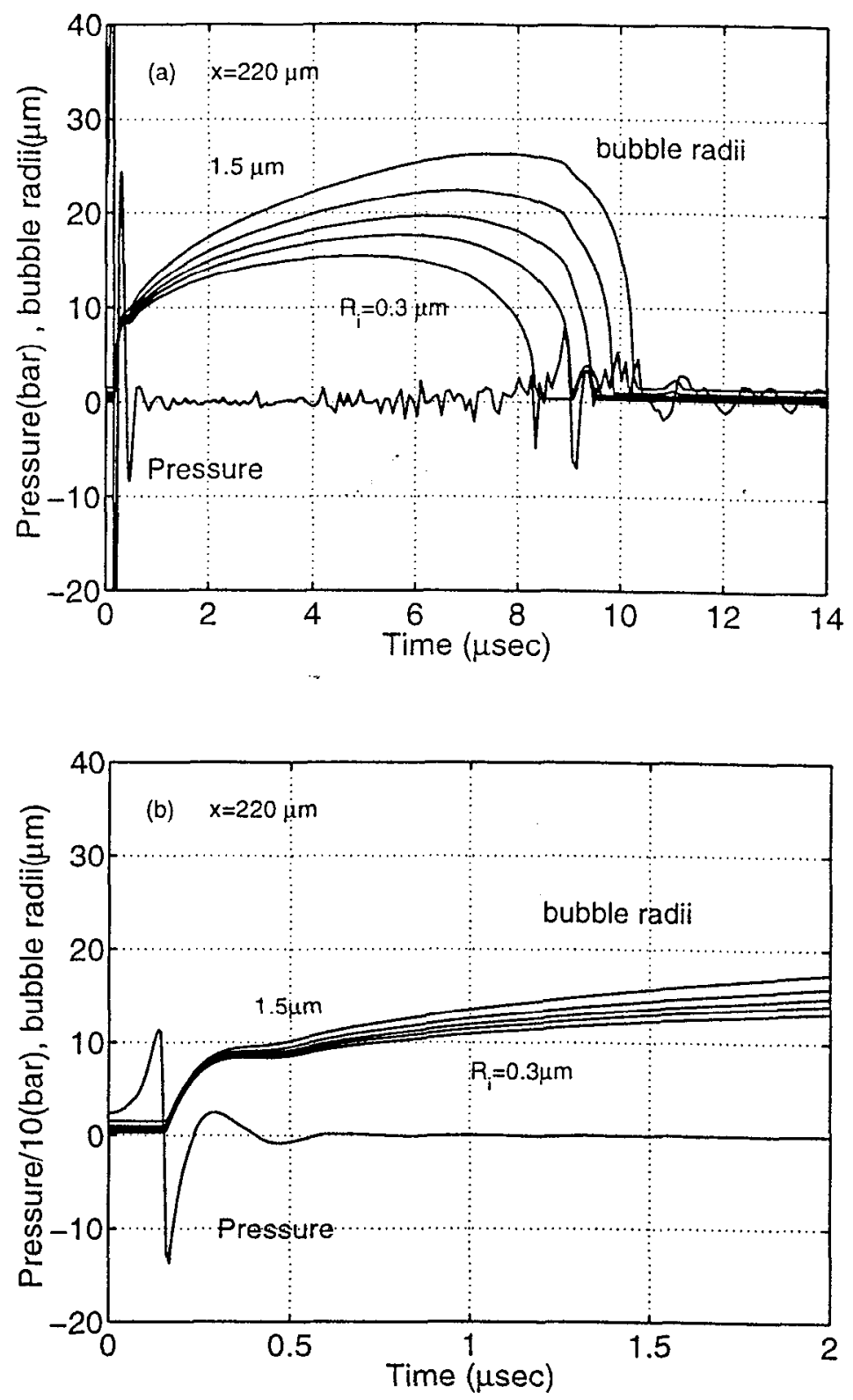

Fig. 6. Same as fig.(5a) but with: (a) pressure in bar and (b) pressure/10 in a more detailed time scale.

Figure (7) shows the bubble lifetimes as a function of distance $\mathrm{x}$ from the free boundary for a laser fluence of $1.53 \mathrm{~J} / \mathrm{cm}^{2}$ and densities $n_{0} \approx 0$ and $10^{6} \mathrm{~cm}^{-3}$. Figure (7) also includes the experimental results obtained in ref.(1). This fluence is consistent with a temperature rise at the free boundary of $\Delta T_{o}=40^{\circ} \mathrm{C}$. Comparing the lifetime results for bubbles with initial radius $R_{i}=1 \mu \mathrm{m}$, for $n_{0} \approx 0$ and $n_{0}=10^{6} \mathrm{~cm}^{-3}$, there is an increase in lifetime from $6 \mu \mathrm{sec}$ to $10 \mu \mathrm{sec}$ for $100<x<350 \mu \mathrm{m}$. It is clear that impurity interactions have a large influence on the bubble lifetime and should be calculated consistently with the hydrodynamic motion. The lifetime increase from the bubble boundary for, $x<100 \mu \mathrm{m}$, where the tensile component is low and is screened by the compression component. At larger distances, $x>350 \mu \mathrm{m}$, the lifetime is again reduced because the tensile component is screened by the void growth. Good agreement is obtained for the bubble lifetimes between our computational results and experimental results of ref.(1) for $n_{0}=10^{6} \mathrm{~cm}^{-3}$. 
Figure (8) considers the impurity density effect on the bubble lifetimes for bubbles with initial radius $R_{i}=1 \mu \mathrm{m}$, for a boundary temperature rise of $\Delta T_{0}=18{ }^{\circ} \mathrm{C}$ and $40^{\circ} \mathrm{C}$ at a distance $x=220 \mu \mathrm{m}$. For the case $\Delta T_{o}=40^{\circ} \mathrm{C}$ there is an increase in bubble lifetime with increasing impurity density because of the delay in reaching the 1 bar ambient pressure as the impurity density increases. At further higher densities, $n_{0}>5 \cdot 10^{6}$, pressure screening and oscillations are strongly increased and the bubble lifetimes are strongly reduced. For smaller $\Delta T_{0}=18{ }^{\circ} \mathrm{C}$, compare to a fluence of $0.68 \mathrm{~J} / \mathrm{cm}^{2}$, the bubble lifetimes are shorter and have a much weaker dependence on the impurity density. The results for $\Delta T_{0}=18{ }^{\circ} \mathrm{C}$ are consistent with the experimental results. ${ }^{1}$

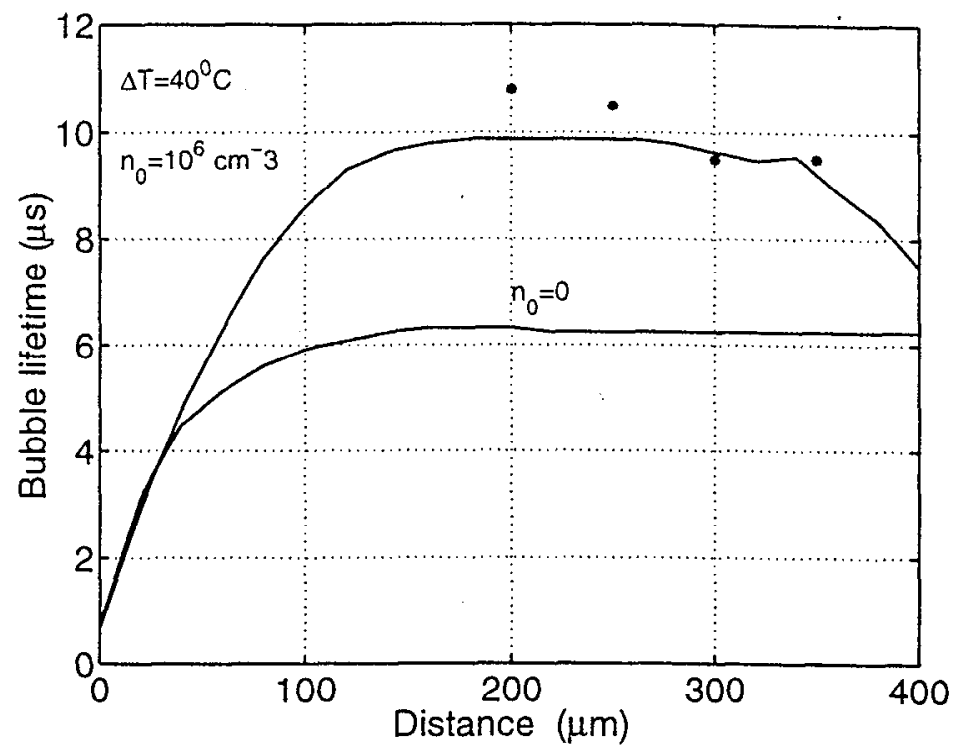

Fig. 7. Bubbles lifetime vs distance $x$ for bubbles with initial radius $R_{i}=1.0 \mu m, n_{0}=10^{6} \mathrm{~cm}^{-3}$ and $n_{0} \approx 0$. The laser fluence is $1.53 \mathrm{~J} / \mathrm{cm}^{2}$ and the temperature rise on system boundary is $\Delta T_{0}=40{ }^{\circ} \mathrm{C}$. The dots are Paltauf et al experimental results ${ }^{1}$. 


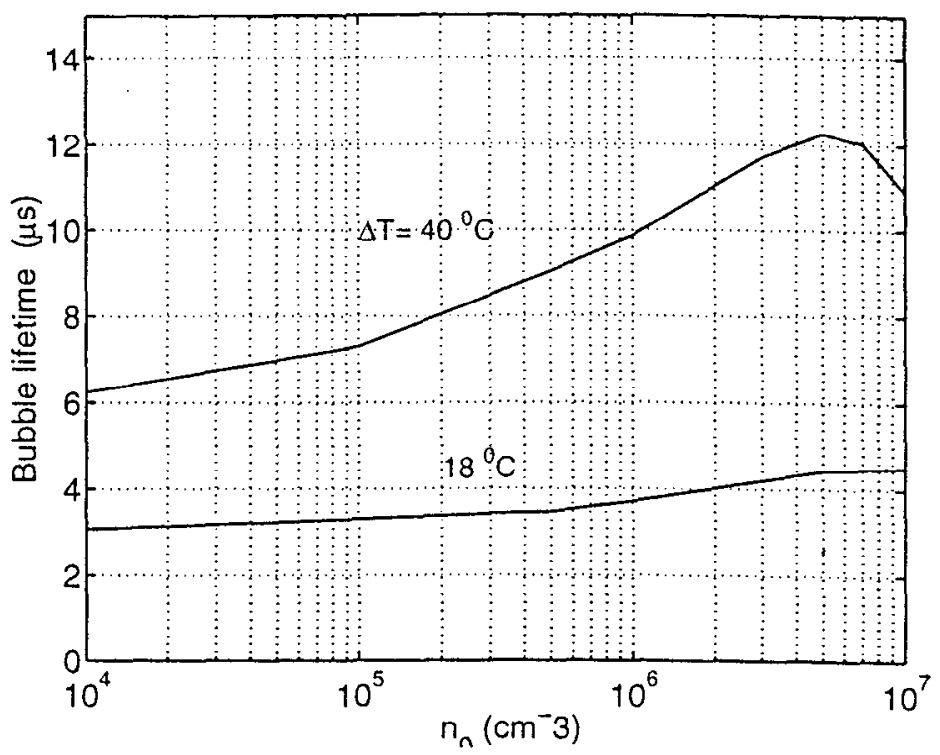

Fig. 8. Bubbles lifetime vs impurity density $n_{0}$ for bubbles with initial radius $R_{i}=1.0 \mu \mathrm{m}$, at a distance $x=220 \mu \mathrm{m}$, boundary temperature rise of $\Delta T_{0}=18{ }^{\circ} \mathrm{C}$ and $40{ }^{\circ} \mathrm{C}$.

\section{CONCLUSIONS}

For moderate impurity densities there is strong interaction between the stress wave and the ensemble of evolving voids on a short time scale of stress wave propagation $(\sim 100 \mathrm{~ns})$. The interaction attenuates the stress wave tensile component and induces pressure oscillations. The effect is stronger as the wave propagates inside the system. For low impurity concentration the growing voids interact with each other on a longer time scale $(\sim 10 \mu \mathrm{s})$ through the change in the pressure field and strongly influence their lifetimes. Thus bubble evolution, long time before coalescence, should be treated self-consistently with the hydrodynamic pressure in the system and can not be treated as non-interacting bubbles as done in many cases. We found that various types of impurities with different initial radii nucleate voids which evolve on a different time scale. Thus real types of impurity distributions should be considered.

The self-consistent effect of stress wave propagation and void evolution should be tested for different physical effects such as viscosity, strength and failure, and in different types of tissues. More experiments should be done with different concentrations of impurities to :verify the interaction between stress wave propagation and bubble evolution.

\section{ACKNOWLEDGMENT}

The work of R. London and P. Amendt was preformed under the auspices of the U. S. Department of Energy by the Lawrence Livermore National Laboratory under Contract No. W7405-Eng-48. 


\section{REFERENCE}

1. G. Paltauf and H. Schmidt-Kloiber, "Microcavity dynamics during laser-induced spallation of liquids and gels", Appl. Phys. A 62 (1996) p.303.

G. Paltauf and H. Schmidt-Kloiber, "Modeling and experimental observation of photomechanical effects in tissue-like media",

Proceedings of Laser Tissue Interaction VI, SPIE 2391, (1995) p.403.

2. A. Oraevsky and S. L. Jacques, "Mechanism of laser ablation for aqueous media irradiated under confined-stress conditions", J. Appl. 78 (1995) p. 1281.

M. Frenz, G. Paltauf and H. Schmidt-Kloiber, Phys. Rev. Lett. 76 (1996) p. 3546, "Laser-generated cavitation in absorbing liquid induced by acoustic diffraction".

3. Celliers, L. Da Silva, N.J. Heredia, B. M. Mammini, R.A. London and M. Strauss, "Dynamic of laser-induced transients produced by nanosecond duration pulses", in Laser in Surgery VI, SPIE 2671 (1996), p.11.

4. D.R. Curran, L. Seaman, D.A. Shockey," Dynamic failure of solids", Physics Report, 147 (1987), p.253.

5. M.E. Glinsky, D. S. Bailey and R. A. London, "LATIS modeling of laser induced midplane and backplane spallation", Proceedings of Laser Tissue Interaction VIII, SPIE 2975, (1997) p.374.

6. Lord Rayleigh, "On the pressure developed in a liquid on the collapse of a spherical cavity", Philos. Mag. 34, (1917) p.94. M. Plesset, "The dynamics of cavitation bubbles", J. Appl. Mech. 16, 277 (1949).

7. R.T. Knapp, J. W. Daily and F. G. Hammitt, Cavitation (McGraw Hill, New York), p.94.

8. A. Vogel, S. Busch and U. Parlitz, "Shock wave emission and cavitation bubble generation by picosecond and nanosecond optical breakdown in water", J. Acoust. Soc. Am., 100 (1996) p. 148.

9. M.E. Glinsky, P. A. Amendt, D. S. Bailey, R. A. London, A. M. Rubenchik and M. Strauss, "Extended Rayleigh model of bubble evolution with material strength compared to detailed dynamic simulations", Proceedings of Laser Tissue Interaction VIII, SPIE 2975, (1997) p.318.

10. P. Amendt, M. Strauss, R. A.London, M. E. Glinsky, D. J. Maitland, P.M. Celliers, S.R. Visuri, D. S.Baily and D. A. Young, "Vapor bubble studies for medical applications: dynamics and energetics", to be published.

11. D. Stauffer and A: Aharony, Introduction to percolation theory,(Taylor and Francis 1992), M. Sahimi, Application of Percolation Theory (Taylor and Francis 1994).

12. L. Haar, J.S. Gallagher and G.S. Kell, NBS/NRC Steam Tables (McGraw-Hill, New York, 1984). 